\title{
Medizin und Heilkunst zwischen Markt- und Geschenkökonomie
}

\author{
Johannes Bircher \\ Dr. med., Professor emeritus Hepatologie, Universitätsklinik für Viszerale Chirurgie und Medizin, Inselspital Bern
}

\begin{abstract}
Marktökonomie beschreibt einen Tausch von Gütern gegen Geld. Wenn sich Menschen innerhalb der Gesundheitsberufe auch als Personen einbringen und durch ihre Beziehungsarbeit zur Linderung von krankhaften Prozessen beitragen, ist eine Bewertung allein nach marktökonomischen Gesichtspunkten nicht stimmig.
\end{abstract}

\section{Résumé}

La médecine, l'art de guérir entre économie de marché et économie du don L'économie de marché, à savoir l'échange de marchandises contre de l'argent, doit clairement être distinguée du don, car celui-ci est soumis à d'autres règles que celles du marché. Les prestations médicales et de soins courantes sont généralement indemnisées par le biais de tarifs et c'est pourquoi elles font partie de l'économie de marché. La relation aux patients peut en revanche parfois être la source d'un engagement extraordinairement créatif dont les effets sont particulièrement bénéfiques à la santé des patients. Les prestations de ce type ne doivent pas être considérées comme des prestations courantes mais plutôt comme des dons. Les activités médicales et de soins ne doivent donc pas uniquement être interprétées et évaluées sur le plan macroéconomique, mais également sur le plan de l'économie du don.

\section{Ausgangslage}

Zwei Aspekte begleiten die gesundheitliche Betreuung von Menschen durch Ärzte und Pflegende. Einerseits sind diese Tätigkeiten Arbeit und andererseits spielen dabei menschliche Beziehungen und Kreativität eine wichtige Rolle. Deshalb können sie nicht einfach nur der Marktökonomie zugeordnet werden. Wenn sich Menschen innerhalb der Gesundheitsberufe auch als Personen einbringen und durch ihre Beziehungsarbeit zur Linderung von krankhaften Prozessen beitragen, ist eine Bewertung allein nach marktökonomischen Gesichtspunkten nicht stimmig. Geschenkökonomie könnte angemessener sein [1]. Doch was würde das bedeuten? Um dieser Frage näherzukommen, müssen die beiden Denkweisen mit der Tätigkeit von Ärzten und Pflegenden zusammengebracht werden.

Grundsätzlich beschreibt die Marktökonomie einen Tausch von Gütern gegen Geld, wobei der Preis durch
Nachfrage, bestimmt wird. Die Metapher der «unsichtbaren Hand» wurde 1776 durch den schottischen Moralphilosophen Adam Smith (1723-1790) geprägt. Er gilt als Begründer der Nationalökonomie. Mit der «unsichtbaren Hand» meinte er, dass eine freie Wirtschaft allen den grössten Nutzen bringt, wenn jeder seinen eigenen Nutzen maximiert. Im Gegensatz dazu bezeichnet die Geschenkökonomie eine wohlgemeinte und freiwillige Übergabe von Gütern oder Werten von einer Person auf eine andere, die ihrerseits die übertragenen Geschenke nicht im engeren Sinne als Eigentum betrachtet. Dieser Vorgang löst weitere Reaktionen aus. Ein Gut wird z.B. weitergegeben und sein Wert hat die Tendenz, sich sogar zu vergrössern. Auf eine unkontrollierbare Art wird es auch irgendwie wieder zum Schenkenden zurückgeführt. Seit der Antike bestehen diese beiden Arten von Ökonomie nebeneinander. Sie haben sich mit der Zeit gewandelt und bis heute fortwährend weiterentwickelt.

\section{Hintergründe zur Marktökonomie}

Die Marktökonomie ist heute die verbreitetste Methode zum Austausch von Gütern. Am einfachsten ist sie im Supermarkt erlebbar. Güter werden in den Gestellen ausgesucht, in den Wagen gelegt und am Schluss an der Kasse bezahlt. Dabei ist es nicht einmal nötig, mit jemandem zu sprechen. Sogar an der Kasse ist der Kontakt von Mensch zu Mensch nur technischer Natur. Ein persönliches Gespräch wäre eher störend. Somit kann der Austausch von Alltagsgütern heute als formeller Vorgang betrachtet werden, bei dem menschliche Beziehungen bedeutungslos oder sogar hinderlich sind.

Zur Marktökonomie gehört auch das Leihen von Geld, z.B. zum Kauf eines Grundstücks oder einer Wohnung. 
Nur ein überhöhter Zins wird als Wucher bezeichnet. Der Zins wird heute auch von der "unsichtbaren Hand" bestimmt. Die Marktökonomie setzt allerdings einen "freien» Markt voraus. Dafür ist Redlichkeit im Einhalten der «Spielregeln» die entscheidende Voraussetzung. Sowohl der Wert von Gütern als auch ihre Preise können zum Schaden Dritter manipuliert und verfälscht werden. Damit wird die «unsichtbare Hand», d.h. der Markt, ausgehebelt. In diesem Sinne können auch Geschenke an Vertreter der Gesundheitsberufe problematisch sein.

\section{Hintergründe zur Geschenkökonomie}

Bei Urvölkern wurde nicht gehandelt. Güter wurden nach Bedarf frei ausgetauscht. Wenn jemand ein Geschenk erhielt, fühlte er sich dem Schenkenden gegenüber verbunden. Das Geschenk durfte gebraucht oder verbraucht werden. Es wurde aber nicht im engeren Sinne zu einem Eigentum des Beschenkten, sondern es entstand eine Verpflichtung, mindestens jemandem etwas Angemessenes weiterzuschenken. Der neu Beschenkte handelte im gleichen Sinne, so dass Geschenke "zirkulierten" und sogar irgendwie, d.h. auf nicht vorhersehbare Weise, wieder auf die Ausgangsperson zurückkamen. Im Rahmen dieser Zirkulation hatte das Geschenk die Tendenz, grösser zu werden. Kam ein Gast zu einem Stamm oder einer Sippe, so wurden ebenfalls Geschenke ausgetauscht. Das Schenken vermehrte das Ansehen des Schenkenden. Diese Kultur des Schenkens war für die Urvölker wichtig, weil sie gemeinschaftsfördernd war. Sie konnte an vielen verschiedenen Stellen in der Welt unabhängig voneinander beobachtet werden.

Betrachten wir das Schenken heute, so stellen wir ähnliche Phänomene fest. Wenn wir schenken oder ein Geschenk erhalten, fühlen wir uns mit den Beschenkten oder Schenkenden verbunden. Ein angenommenes Geschenk verpflichtet uns auf irgendeine Art und Weise. Wir müssen uns mit dem Geschenk auseinandersetzen und spüren ein Bedürfnis, ebenfalls irgendetwas ungefähr Gleichwertiges zu schenken. Auf diese Weise haben Geschenke auch bei uns eine natürliche Tendenz, grösser zu werden.

Früher gab es auch Geschenke der Natur. Ihre Fruchtbarkeit war für die Menschen ein riesiges Geschenk. Der Apfelbaum z.B. gab Äpfel, die Kuh Milch und das Huhn Eier. Deshalb haben sich Urvölker mit verschiedenen Riten dafür bedankt. Auch bei uns wurde noch vor nicht langer Zeit eine "Sichlete», d.h. ein Erntedankfest, abgehalten. Das Unverkäufliche eines Geschenks kommt in diesen Bräuchen gut zum Ausdruck. Doch heute sind Nahrungsmittel und Rohstoffe zu Handelswaren geworden. Das ist ein wesentlicher Kulturwandel von der Geschenkökonomie zur Marktökonomie. Der Geschenkcharakter wird aber heute noch empfunden, wenn ein Kind zur Welt kommt. Das neue Leben ist für die Eltern ein riesiges Geschenk, das sie zwar verpflichtet, aber auch fortwährend beschenkt. Diese Beispiele mögen genügen, um die Geschenkökonomie und ihren Wandel mit der Zeit zu illustrieren.

Kreative Schöpfungen, z.B. von Künstlern, sind auch Geschenke. Bis heute werden sie nicht adäquat bezahlt. Vincent van Gogh (1853-189o) z.B. konnte auf dem Markt kein einziges Bild verkaufen, obwohl heute seine Werke auf Auktionen mehrere Millionen Wert sind. Viele Künstler mussten dauernd oder mindestens so lange in Armut leben, bis sie genügend bekannt wurden. Kreative Werke werden nicht gefertigt wie Tische und Stühle. Meist müssen sie trotz des Könnens des Künstlers irgendwie erduldet und erlitten werden. Der schöpferische Vorgang ihrer Erschaffung ist oft mühsam und das Resultat unsicher. Viele Künstler betrachten ihre eigenen Werke als empfangene Geschenke. Die schlechte Bezahlung lässt darauf schliessen, dass auch die Gesellschaft sie eher wie Geschenke behandelt. Es erhebt sich deshalb die Frage, ob gut bezahlte Kunstwerke noch der Geschenkökonomie entsprechen.

\section{Beziehungen zwischen Geschenk- und Marktökonomie}

In der Urgeschichte war vermutlich die Geschenkökonomie vorherrschend. Seit der Zeit von Moses (13. Jahrhundert vor Christus) bestehen beide Ökonomieformen nebeneinander. Im Alten Testament wird beschrieben, wie Güter innerhalb der Gemeinschaft frei auszutauschen sind. Im Gegensatz dazu durfte im Handel mit Fremden ein Preis und wenn nötig auch ein Zins verlangt werden [2]. Geld in Form von

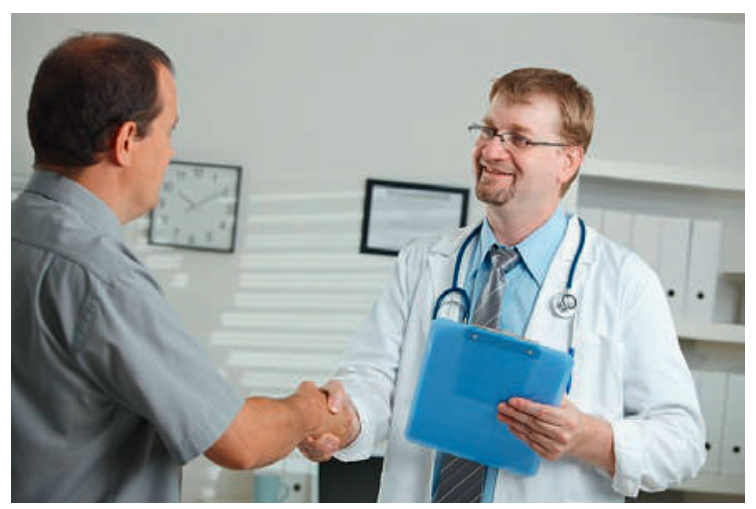

Die Arzt-Patienten-Beziehung: mehr als ein Tauschgeschäft von Leistung gegen Geld. 
Münzen wurde erstmals ungefähr 650 vor Christus in Lydien, d.h. in der heutigen Türkei, geprägt und erleichterte den Handel.

Auch im Mittelalter herrschte vorwiegend eine $\mathrm{Ge}$ schenkökonomie. Gewöhnliche Bürger durften keine Zinsen nehmen. Das war nur den Juden vorbehalten, die auch durchaus Wucherzinse fordern durften. Erst während der Reformation begann sich die Marktökonomie durchzusetzen. Unter starkem Druck erlaubte Luther einen Zins von 5 oder höchstens 8 Prozent [3]. Dadurch konnte sich die Marktökonomie ausbreiten, und die Geschenkökonomie wurde auf immer engere Kreise reduziert, z.B. auf Familie und nahe Freunde. Heute ist die Marktökonomie weit im Vordergrund. Das hat den Vorteil, dass sich der Handel im Alltag effizient vollziehen kann, ohne dass menschliche Beziehungen bemüht werden müssen. Zudem wird der Unterschied zum Schenken, d.h. der Beziehungsaspekt, deutlich erlebbar. Interessant ist auch, dass z.B. Nahrungsmittel, die früher als Geschenke galten, heute zu Konsumprodukten wurden. Erdöl, Mineralien und andere Rohstoffe sind ebenfalls zu Handelswaren geworden, mit denen sich gewisse Kreise maximal bereichern konnten. Die Idee, dass die Natur uns Geschenke zur Verfügung stellt, ist somit in den Hintergrund getreten. Dafür sind kreative und karitative Prozesse weitgehend bei der Geschenkökonomie geblieben, doch nicht ganz. Spendenaufrufe z.B. sind in den letzten Jahren trickreich "professionalisiert» worden. Mit der Bitte, Geld zu spenden, wird ein kleines Geschenk verschickt, um den Empfänger dieses Geschenks im Sinne der Geschenkökonomie zu verpflichten.

Diese Beispiele zeigen, dass zwischen der Geschenkökonomie und der Marktökonomie Wechselbeziehungen bestehen, die sich als Folge kultureller und historischer Entwicklungen verändern. Gewisse Vorgänge wechseln von einer Art der Ökonomie zur anderen.

\section{Leistungen für die Gesundheit zwischen Markt- und Geschenkökonomie}

In der Medizin hat sich auch ein Wandel von der Geschenkökonomie zur Marktökonomie vollzogen. Zwar stellten die Ärzte schon seit langem Rechnungen, wie das z.B. Molière im «Eingebildeten Kranken" beschrieb. Die Pflege gehörte aber klar zur Geschenkökonomie. Das drückte sich z.B. in der Bezeichnung «Krankenschwester» aus, die heute im sinne der Marktökonomie zur «Pflegefachfrau» geworden ist. Es ist deshalb sinnvoll, die Frage neu zu betrachten, welcher Art von Ökonomie Leistungen für die Ge- sundheit zugeordnet werden sollen. Wenn Ärzte ihre Patienten wirklich gut betreuen, d.h. wenn sie ihre Patienten korrekt untersuchen, die angemessenen Tests verordnen, diese zu einwandfreien Diagnosen verarbeiten und die sinnvollen Therapien durchführen, haben sie Leistungen vollbracht, die alle nach Tarif abgerechnet werden. Somit wird ihr Wissen und Können entsprechend der Marktökonomie vergütet. Analoge Überlegungen gelten auch für die anderen Gesundheitsberufe. Im Gegensatz zur reinen Marktökonomie geht eine gesundheitliche Betreuung mit einer menschlichen Beziehung einher. In diesem Sinne bleibt auch heute der Begriff «Patient» eher angemessen als die Bezeichnung Klient.

Vielen Ärzten gelingt es von Zeit zu Zeit, durch eine engagierte und vertiefte Auseinandersetzung mit einem Kranken und durch einen besonderen kreativen Einsatz etwas Ausserordentliches zu realisieren, das dem Patienten zu einer heilenden Perspektive verhilft. Ein solches Ereignis ist am ehesten mit künstlerischem Schaffen zu vergleichen. Dies gilt analog auch für andere Gesundheitsberufe. Wie soll eine solche Leistung honoriert werden? Meines Erachtens ist diese Form der ärztlichen Tätigkeit der Geschenkökonomie zuzuordnen, die nicht mit Geld abgegolten werden kann. In diesem Fall ist eine besondere, gesundheitlich wirksame Arzt-Patienten-Beziehung entstanden. Das heisst, das Geschenk des Arztes führt beim Patienten zu einer menschlichen Verbindung mit ihm und zu einer inneren Verpflichtung, das Geschenkte irgendwie weiterzugeben, bis es schliesslich auf einem unsichtbaren Weg und nicht materiell wieder zum betreffenden Arzt zurückkommt. Mindestens erlebt der Arzt eine besondere Hochachtung. Oft haben auf diese Weise beschenkte Patienten auch den Wunsch, sich beim Arzt mit einem persönlichen Geschenk zu bedanken. Wie damit umgegangen werden soll, ist in jedem einzelnen Fall zu bedenken.

Somit sind heute die meisten Leistungen von Ärzten und Pflegefachpersonen der Marktökonomie zuzuordnen. Bei uns werden die Preise nicht von der unsichtbaren Hand bestimmt, sondern durch Tarife festgesetzt. Die Arzt-Patienten-Beziehung und besondere kreative Einsätze für die Gesundheit einzelner Personen müssen am ehesten als Geschenkökonomie verstanden und entsprechend gehandhabt werden. Es ist wichtig, diese Unterschiede zu erkennen und zu reflektieren.

\footnotetext{
Literatur

1 Hyde L. The gift, creativity and the artist in the modern world. New York: Vintage Books, Random House; 2007

2 . Mose, Kapitel 23, Verse 19 und 20.

3 Hyde L. [1] Seiten $156 \mathrm{ff}$.
} 